\title{
LAS RESERVAS A LOS TRATADOS DE DERECHOS HUMANOS EN MÉXICO: ESTADO DE LA CUESTIÓN*
}

\section{Human Rights Treaties Reservations in Mexico: the State of the Art}

Recibido: 2 de abril de 2020

Aceptado: 1 de julio de 2020

Alicia María Pastor García

Doctoranda en Derecho Internacional Público y

Relaciones Internacionales

Universidad de Málaga

ampastor@uma.es

\section{RESUMEN}

En el presente artículo se plantea la importancia que tienen las reservas que formulan los Estados a los Convenios internacionales sobre Derechos Humanos y cómo esas reservas restringen las obligaciones asumidas por los Estados en este ámbito. En concreto, se trata de examinar la práctica convencional de México en materia de reservas, como medio para determinar la posición de este Estado respecto de la salvaguardia de los Derechos Humanos que se contienen en estos Tratados internacionales.

\section{PALABRAS CLAVES}

México, objeciones, reservas, Tratados de Derechos Humanos.

\begin{abstract}
In this article we considered the importance of the reservations that the States formulate to the international Human Rights Treaties and how those reservations restrict the obligations assumed by States in this area. In particular, it is about examining the conventional practice of Mexico regarding reservations, as a means to determine the position of this State regarding the safeguarding of Human Rights contained in these international Treaties.
\end{abstract}

\section{KEYWORDS}

Mexico, objections, reservations, Human Rights Treaties.

\footnotetext{
* El presente trabajo se enmarca en el Proyecto de Investigación SEJ-405 “Cooperación Judicial en el ámbito Penal", financiado por la Junta de Andalucía.

Los resultados de esta investigación fueron expuestos por la autora en el IV Coloquio Binacional México-España: "El Derecho en el análisis de casos: conflictos, razonamientos y decisiones", que tuvo lugar en Málaga, del 13 al 15 de diciembre de 2017.
} 
Sumario: 1. Consideraciones previas. 2. Análisis de la práctica convencional de México. 2.1. Reservas. 2.2. Objeciones. 3. Otros elementos para evaluar el compromiso internacional de México respecto de la protección de los Derechos Humanos. 4. Conclusiones. Bibliografía.

\section{Consideraciones previas.}

La creciente preocupación por la protección de los Derechos Humanos en la comunidad internacional ha llevado a la paulatina elaboración de numerosos instrumentos jurídicos internacionales auspiciados por las Naciones Unidas, como medida para garantizar la defensa de los Derechos inherentes al ser humano con independencia de su nacionalidad o de la jurisdicción del Estado en el que se encuentra. Así pues, el alcance universal de estos convenios multilaterales es uno de los objetivos nucleares en el seno de esta Organización Internacional, puesto que las obligaciones contraídas por un Estado al ratificar un instrumento convencional cuyo objeto es la protección de los Derechos Humanos no sólo tienen relevancia para su ordenamiento interno, sino que afectan de forma directa a la promoción y defensa de los mismos en el Derecho Internacional.

Sin embargo, la asunción de obligaciones internacionales en materia de Derechos Humanos mediante la ratificación de convenciones ${ }^{1}$ da lugar, en muchos casos, a la formulación de reservas por parte de los Estados, con el fin de excluir o modificar los efectos jurídicos de ciertas disposiciones de ese tratado en su aplicación al Estado Parte que la formula ${ }^{2}$.

Por esta razón, el número de convenios ratificados por parte de un país no es el único medio para comprobar su compromiso con la salvaguardia de los derechos que en ellos se contienen, sino que habrá que atender también al alcance que ha tenido esta aceptación del tratado internacional. De este modo, el examen de las reservas formuladas por un país a determinadas convenciones multilaterales sobre Derechos Humanos, así como de las objeciones a las reservas de otros Estados Partes, se erigen en elementos idóneos para determinar la implicación de ese Estado con la protección efectiva de los Derechos Humanos.

El presente artículo se centrará en el análisis de la práctica convencional de México en materia de Derechos Humanos para tratar de obtener conclusiones relativas a la posición de este Estado acerca de una cuestión de gran trascendencia internacional, si bien este fundamento no deja de ser indiciario en lo que respecta al cumplimiento efectivo de las obligaciones convencionales asumidas por parte de este país ${ }^{3}$. El interés que reviste

\footnotetext{
${ }^{1} \mathrm{O}$ cualquier otra forma de expresión del consentimiento del Estado en obligarse por ese tratado. De hecho, las reservas pueden hacerse en el momento de la firma, ratificación, aceptación, aprobación del tratado o su adhesión al mismo.

${ }^{2}$ El Convenio de Viena sobre el Derecho de los Tratados, adoptado en Viena el 23 de mayo de 1969, define la reserva en su artículo 2.1.D) como "una declaración unilateral, cualquiera que sea su enunciado o denominación, hecha por un Estado al firmar, ratificar, aceptar o aprobar un tratado o al adherirse a él, con el objeto de excluir o modificar los efectos jurídicos de ciertas disposiciones del tratado en su aplicación a ese Estado".

${ }^{3}$ En este sentido, vid. HATHAWAY, O.A., (2002) “Do Human Rights Treaties Make a Difference?", The Yale Law Yournal, Faculty Scholarship Series, Vol. 111, Paper 839: pp. 1935-2042, que cuestiona que exista una efectiva correlación entre la ratificación de los tratados de derechos humanos y las prácticas de derechos humanos por parte de ese Estado. En palabras de este autor: "External pressure on countries to demonstrate a commitment to human rights norms creates strong incentives for countries to engage in favorable expressive behavior by ratifying human rights treaties. But (...), there is little incentive for
} 
en nuestra opinión la práctica de este Estado deriva del lugar destacado que ocupa en la comunidad iberoamericana y su participación en numerosos órganos de protección de Derechos Humanos. De ahí que consideremos que el análisis que se lleva a cabo en esta publicación puede aportar elementos relevantes en el estudio de la práctica convencional sobre Derechos Humanos extrapolable y representativa de la práctica internacional desarrollada por los Estados que conforman este ámbito geográfico.

Estas consideraciones nos llevan, asimismo, a centrarnos en la práctica convencional de México en materia de reservas respecto de tratados internacionales sobre Derechos Humanos creados bajo los auspicios de Naciones Unidas.

Al respecto, dado que la reserva es una declaración unilateral ligada a la manifestación del consentimiento de un Estado en ser parte del convenio ${ }^{4}$, cuyo propósito es limitar o modificar los efectos jurídicos que para sí tendrá la disposición del tratado a la que la misma se refiera, podemos deducir que la inclusión de este tipo de declaración por parte de un Estado podría, en determinados casos, comprometer la integridad del instrumento convencional y la debida protección de los derechos que en él se consagran por parte de este sujeto de Derecho Internacional ${ }^{5}$.

Además, hemos de tener en cuenta que las reservas plantean difíciles cuestiones técnicas de gran importancia jurídica ${ }^{6}$ puesto que, en palabras de CHAUMONT, "le procédé contemporain le plus important pour diversifier les engagements à l'intérieur d'un même traité multilatéralest celui des réserves"7. Tanto es así, que el tema de las reservas a los tratados ha ocupado la agenda de la Comisión de Derecho Internacional de Naciones Unidas (en adelante, CDI) durante un largo período de tiempo, que ha concluido con la redacción de "la Guía de la Práctica sobre las Reservas a los Tratados"8 que da respuesta a muchas de las lagunas que presenta el régimen jurídico establecido en la Convención de Viena sobre el Derecho de los Tratados (en adelante, CVDT), de 1969, relativo a las reservas.

Una de las cuestiones que suscita el examen de las reservas es que su determinación puede representar un reto a niveles prácticos, ya que es frecuente encontrar reservas bajo la denominación de otras figuras jurídicas que carecen de una definición convencional,

\footnotetext{
ratifying countries to make the costly changes in actual policy that would be necessary to meet their treaty commitments", p. 2020.

${ }^{4}$ Sobre el carácter unilateral de las reservas vid. QUEL LÓPEZ, J. (1991), Las reservas a los tratados internacionales (un examen de la práctica española). Bilbao: Servicio Editorial de la Universidad del País Vasco, pp. 23-24.

${ }^{5}$ Puesto que “(...) les Etats contractants peuvent moduler comme ils l'entendent, et à peu près sans limites, le jeu des réserves et des objections, soit dans le traité lui-même, soit, si celui-ci est muet à cet égard, par le moyen des actes unilatéraux", CARRILLO SALCEDO, J.A. (1996), "Cours général de Droit International Public: Droit International et Souveraineté des Etats", Recueil des Cours de l'Académie de Droit International, vol. 257: pp. 217, p. 65.

${ }^{6}$ Como deja claro el Relator Especial, PELLET, A. (2013), "The ILC Guide to Practice on Reservations to Treaties: A General Presentation by the Special Rapporteur", The European Journal of International Law, vol. 24, nº 4: pp. 1061-1097, p. 1063.

${ }^{7}$ Vid., CHAUMONT, C. (1970), “Cours général de droit international public”, Recueil des Cours, vol. 129: pp. 333-528, p. 446.

${ }^{8}$ El texto de esta Guía de la Práctica fue aprobado por la CDI en su $63^{\circ}$ período de sesiones, del año 2011. Vid., Texto de la Guía de la Práctica y los comentarios, Informe de la Comisión de Derecho Internacional A/66/10/Add.1, Documento de la Asamblea General de las Naciones Unidas, sexagésimo sexto período de sesiones, suplemento núm. 10.
} 
como es el caso de las declaraciones interpretativas ${ }^{9}$. No obstante, este ha sido uno de los vacíos convencionales colmados por la CDI en su trabajo, que se ha ocupado de proporcionar un concepto de declaración interpretativa, en los siguientes términos: "una declaración unilateral, cualquiera que sea su enunciado o denominación, hecha por un Estado o por una Organización Internacional con el objeto de precisar o aclarar el sentido o el alcance de un tratado o de algunas de sus disposiciones" 10 . Es decir, establecer una determinada interpretación de esa disposición convencional. En consecuencia, no deben asimilarse los efectos de ambas figuras jurídicas, puesto que, como bien apunta HORN "the legal effect of certain provisions must be comprehended as the criterion of a norm which imposes obligations and confers rights. Now, the "excluding effects" of a reservation is to be read as "negating the force of a norm", a duty for example is turned in liberty. An interpretative declaration qua interpretation has not such effect. Such a declaration has the function to assert the existence and contents of a norm where the text of the treaty leaves us in a doubt. it is not intented to have a derogatory effect, which in the case with reservations" 11 .

Aunque se trata de conceptos diferenciados en teoría, lo cierto es que la práctica de los Estados continúa siendo poco adecuada a esta distinción, y resulta frecuente encontrar auténticas reservas, por parte de muchos Estados, bajo la denominación "más amable" de declaración interpretativa ${ }^{12}$. Una situación de la que también se ha hecho eco la CDI y a la que trata de ofrecer una solución que ha cristalizado en su Guía de la Práctica al establecer que "la calificación de una declaración unilateral como reserva o declaración interpretativa está determinada por los efectos jurídicos que su autor se propone producir" 13 , de manera que habrá que atender al contenido de la misma ${ }^{14}$, sin que resulte vinculante la denominación proporcionada por el Estado que la formula ${ }^{15}$.

Otro de los aspectos más controvertidos en relación con las reservas se refiere precisamente a los criterios de validez de las mismas y, concretamente,

"respecto del peso que cabe atribuir, respectivamente, al criterio de la compatibilidad de la reserva con el objeto y fin del tratado, por un lado, y por el otro,

\footnotetext{
${ }^{9}$ En palabras de RIQUELME CORTADO: "El núcleo duro de las omisiones que dejó subsistir la Convención de Viena de 1969 tiene que ver con la definición y el régimen jurídico de las declaraciones interpretativas". Vid. RIQUELME CORTADO, R. (2004), Las reservas a los tratados: Lagunas y ambigüedades del Régimen de Viena. Murcia: Universidad de Murcia, Servicio de Publicaciones, Colección Estudios de Derecho, p. 33.

${ }^{10}$ Guía de la práctica de la CDI, apartado 1.2. Sin embargo, las declaraciones interpretativas siguen sin contar con una definición internacional, puesto que la guía de la CDI no es más que un instrumento que ha adoptado "la forma de un proyecto de artículos cuyas disposiciones, junto con comentarios, constituirían directrices para la práctica de los Estados y de las organizaciones internacionales en materia de reservas", vid., Informe de la Comisión de Derecho Internacional sobre la labor realizada en su $47^{\circ}$ período de sesiones, 1995, Doc. A/50/10, Capítulo VI, p. 111, párr. 487 b).

${ }^{11}$ Vid., HORN, F. (1988), Reservations and Interpretative Declarations To Multilateral Treaties, La Haya: T.M.C. Asser Instituut, Institute for International and European Law, pp. 237-238.

${ }^{12}$ Puesto que la figura jurídica de la declaración interpretativa cuenta con una mayor aceptación a nivel internacional.

${ }^{13}$ Guía de la práctica, artículo 1.3. relativo a la distinción entre las reservas y las declaraciones interpretativas.

${ }^{14}$ Atendiendo al sentido de sus palabras, interpretadas de buena fe. Art. 1.3.1. de la Guía.

15 "La denominación que se dé a una declaración unilateral formulada a un tratado no prejuzga en uno u otro sentido su condición de reserva (art. 2.1.d CV). Es el efecto que la declaración persigue, y no el nombre que reciba, lo que determina su naturaleza y régimen jurídico.” REMIRO BROTONS, A. et al. (2010), Derecho internacional: curso general. Valencia: Tirant lo Blanch, p.289.
} 
a la expresión de los Estados Partes de su postura respecto de la reserva a través de las aceptaciones o las objeciones. (...) lo que causa dificultades a los Estados y a las organizaciones internacionales, en particular (aunque no exclusivamente) en su condición de depositarios"16.

Una cuestión cada vez más importante que se agrava a medida que se incrementan en la práctica internacional las reservas que parte de la doctrina califica como abusivas, esto es, cuya redacción es vaga y de alcance general ${ }^{17}$.

En efecto, el régimen jurídico de las reservas adolece de una serie de ambigüedades que pueden afectar a la asunción de obligaciones convencionales por los Estados en la medida en que restringen el alcance jurídico de las disposiciones objeto de reserva ${ }^{18}$.

Por consiguiente, y tomando en consideración todos los motivos enumerados $u t$ supra, todas estas cuestiones serán examinadas para tratar de determinar el compromiso internacional de México respecto de la protección de los Derechos Humanos que se consagran en estos instrumentos internacionales.

A tenor de estas consideraciones, este trabajo se estructura en tres grandes apartados, en el primero de los cuales se realizará un análisis de la práctica convencional de México en relación con tratados internacionales de Derechos Humanos, cuestión central para determinar el compromiso internacional de este país en esta materia. Este apartado se divide a su vez en dos subepígrafes, el primero de los cuales estará dedicado al estudio de la validez de las reservas y declaraciones interpretativas formuladas por este Estado a los tratados internacionales de Derechos Humanos creados bajo la égida de Naciones Unidas, y el segundo de ellos al análisis de las objeciones de México a reservas formuladas por otros Estados Partes en estos convenios.

En el segundo apartado de este artículo se examinarán otros elementos que nos permitan alcanzar conclusiones relativas al compromiso internacional de México respecto de la protección de los Derechos Humanos, en concreto la aceptación de la competencia de aquellos Comités de creación convencional encargados de supervisar la correcta aplicación de esos tratados internacionales de Derechos Humanos, así como las reservas formuladas en el momento de su ratificación a estos protocolos facultativos.

En el último apartado, procederemos a mostrar las conclusiones alcanzadas en el presente estudio. Se trata de cuestiones no exentas de dificultades que en muchos casos pueden afectar al alcance jurídico de estas convenciones y, por ende, a la adecuada aplicación de las normas internacionales que tienen por objeto garantizar y proteger los derechos del ser humano, de las que a continuación nos ocupamos.

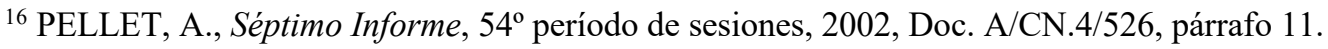

${ }^{17}$ Vid., por todos, RIQUELME CORTADO, R. (2004), Las reservas..., op. cit., pp. 15-16. O "across-theboard reservation", en referencia a Zemanek. Vid. disposición 3.1.5.2. de la Guía de la Práctica, que se refiere a las reservas vagas o generales, estableciendo que "Una reserva habrá de redactarse en términos que permitan percibir su sentido, a fin de determinar, en particular, su compatibilidad con el objeto y el fin del tratado".

${ }^{18}$ De acuerdo con IMBERT: "une réserve est une déclaration unilatérale par laquelle un Etat restreint ses obligations conventionnelles" Vid. IMBERT, P.-H. (1979), Les réserves aux traités multilatéraux. Evolution du droit et de la practique depuis l'avis consultatif donné par la Cour internationale de Justice le 28 mai 1951. Paris: Editions A. Pedone, p.14
} 


\section{Análisis de la práctica convencional de México.}

A tenor de lo expuesto en el anterior epígrafe, vamos a analizar la ratificación por parte de México de aquellos convenios multilaterales de ámbito universal sobre Derechos Humanos depositados ante el Secretario General de las Naciones Unidas ${ }^{19}$, así como, en particular, aquellas reservas o declaraciones interpretativas formuladas por este país en el momento de obligarse por estos tratados internacionales y, en su caso, las reservas formuladas por terceros Estados que han suscitado objeciones por parte de México $^{20}$.

En este contexto, nos hemos ocupado de estudiar aquellos Convenios que, con un marcado carácter sectorial -por establecer de forma concreta la protección de determinados derechos o colectivos, o prohibir y sancionar determinadas conductas-, se dedican a la protección de Derechos Humanos a nivel universal. Dentro de este sistema de protección de Derechos Humanos de ámbito universal auspiciado por Naciones Unidas, encontramos la Convención para la prevención y sanción del delito de genocidio, la Convención internacional sobre la eliminación de todas las formas de discriminación racial, la Convención sobre la eliminación de todas las formas de discriminación contra la mujer, la Convención sobre los derechos del niño o la Convención sobre los derechos de las personas con discapacidad, entre otros muchos.

\subsection{Reservas.}

México ha ratificado la totalidad de los tratados universales de Derechos Humanos cuya conclusión ha auspiciado Naciones Unidas ${ }^{21}$, de manera que el primer elemento de juicio que nos permita evaluar la práctica convencional del Estado mexicano sobre este particular nos lleva a considerar que se trata de una posición favorable hacia la asunción de obligaciones internacionales relativas a la protección de los Derechos Humanos.

Aunque, como hemos dicho con carácter previo, este no representa el único elemento a valorar para alcanzar conclusiones al respecto ya que resulta igualmente significativo, para conocer el alcance de su compromiso jurídico internacional, el estudio de las reservas formuladas por este país. También el análisis de su contenido con el propósito de determinar si las mismas cumplen con los criterios de admisibilidad, si bien la consideración de validez o invalidez de las reservas es una de las cuestiones más controvertidas en este ámbito ${ }^{22}$ a la luz de los artículos 19 y 20 del Convenio de Viena. No obstante, contamos para llevar a cabo este examen con elementos que nos permiten evaluar, aunque sea de forma indiciaria, la validez de las reservas: por un lado las

\footnotetext{
${ }^{19}$ Puesto que en un trabajo de estas características nos vemos en la obligación de acotar el objeto de estudio, no entraremos en el análisis de la práctica de México en relación con otros convenios de Derechos Humanos de ámbito regional, en los que este Estado es parte.

${ }^{20} \mathrm{Si}$ bien se trata de un análisis cuyo estudio exhaustivo reviste una gran complejidad puesto que "The law of treaties with regard to reservations is, in our experience, one of the hardest areas of law to grasp, teach, or condense and simplify without loss of accuracy", en palabras de MILANOVIC, M. y SICILIANOS, L.A. (2013), "Reservations to traties: An Introduction", The European Journal of International Law, vol. 24, no 4: pp. 1055-1059, p. 1055.

${ }^{21}$ Al conjunto de estos tratados ratificados por México podemos acceder en el Diario Oficial de la Federación, en concreto en http://www.ordenjuridico.gob.mx/derechos_humanos.php, [fecha de la consulta, al igual que para los restantes vínculos web que figuran en este artículo, 14 de enero de 2020]. Igualmente, aunque escapa del ámbito de estudio del presente artículo, podemos comprobar que México también ha ratificado numerosos Convenios de Derechos Humanos de ámbito regional, como es la Convención Americana sobre Derechos Humanos -o Pacto de San José-.

${ }^{22}$ Tanto es así que la cuestión terminológica ya planteaba problemas a la hora de calificar a este tipo de reservas como ilícitas o inválidas, siendo finalmente este segundo término el adoptado por la CDI. Vid., RIQUELME CORTADO, R. (2004), Las reservas..., op. cit., pp. 74-82.
} 
objeciones a una reserva por parte de terceros Estados (elemento intersubjetivo), y por otro, la opinión de los órganos de control, encargados de supervisar la correcta aplicación y cumplimiento de las obligaciones convencionales asumidas por los Estados Partes (elemento objetivo) $)^{23}$.

Del mismo modo, resulta controvertida la determinación de los elementos de las reservas que son contrarias al objeto y fin del tratado ${ }^{24}$-imprescindible como criterio de validez de las mismas-, así como las consecuencias jurídicas de la formulación de una reserva de esas características. Afortunadamente, la CDI se ha ocupado de esta materia al establecer ciertos criterios que permiten detectar cuándo una reserva puede ser incompatible con el objeto y fin del tratado ${ }^{25}$. Sin ánimo de profundizar en la cuestión, en primer lugar, la Guía de la Práctica determina qué debe entenderse por "objeto y fin", al que se refiere como "un elemento esencial del tratado, necesario para su estructura general, de tal manera que comprometa la razón de ser del tratado"26. Asimismo, hace referencia a las reservas vagas y generales, puesto que no será posible evaluar su compatibilidad si la reserva en cuestión está redactada en términos que no permitan determinar su alcance. Lo mismo ocurre con determinadas reservas que hacen referencia a su derecho interno ${ }^{27}$.

En el caso de México, a pesar del amplio número de ratificaciones a los tratados de Derechos Humanos, es importante señalar en primer lugar, que no destaca por la formulación de un gran número de reservas o declaraciones que limiten el compromiso convencional asumido por parte de este Estado. Sin embargo esto no quiere decir que México no haya formulado reservas o declaraciones interpretativas, tal y como lo hizo al Pacto internacional de derechos civiles y políticos en el momento de depositar su instrumento de adhesión. En concreto, la reserva que México formuló en su momento se refería a los artículos $13^{28}$ y 25 , inciso b) ${ }^{29}$ del Pacto y basaba las reservas a estas disposiciones convencionales en su propio Derecho interno:

\footnotetext{
${ }^{23}$ Para profundizar sobre esta cuestión, Vid. MARÍN AÍS, J.R. (2015), "Reservas incompatibles con el objeto y fin de la convención para la eliminación de todas las formas de discriminación contra la mujer. El caso de los estados islámicos", Revista Electrónica de Estudios Internacionales, núm. 29: pp. 63, p. 21.

${ }^{24}$ Artículo 19 del CVDT.

${ }^{25}$ Ya en su primer informe PELLET se ocupaba de esta controvertida cuestión. Vid., PELLET, A., "Primer informe sobre la ley y la práctica en materia de reservas a los tratados", Documento A/CN.4/470, pár. 38 y ss.

${ }^{26}$ Directriz 3.1.5.1, dejando claro que el objeto y el fin del tratado deben determinarse de buena fe.

${ }^{27}$ Pues, a pesar de ser una reserva clara y precisa, esta invocación del derecho interno está prohibida en el Convenio de Viena, artículo 27.

${ }^{28}$ Este artículo establece que "El extranjero que se halle legalmente en el territorio de un Estado Parte en el presente Pacto sólo podrá ser expulsado de él en cumplimiento de una decisión adoptada conforme a la ley; $\mathrm{y}$, a menos que razones imperiosas de seguridad nacional se opongan a ello, se permitirá a tal extranjero exponer las razones que lo asistan en contra de su expulsión, así como someter su caso a revisión ante la autoridad competente o bien ante la persona o personas designadas especialmente por dicha autoridad competente, y hacerse representar con tal fin ante ellas". Vid. Pacto Internacional de Derechos Civiles y Políticos, adoptado en Nueva York el 16 de diciembre de 1966, adhesión de México de 24 de marzo de 1981, publicado en Diario Oficial de la Federación (DOF), Decreto Promulgatorio DO 20 de mayo de 1981.

${ }^{29}$ Que dice que todos los ciudadanos tendrán derecho a "votar y ser elegidos en elecciones periódicas, auténticas, realizadas por sufragio universal e igual y por voto secreto que garantice la libre expresión de la voluntad de los electores". Diario Oficial de la Federación (DOF), Decreto Promulgatorio DO 20 de mayo de 1981.
} 
“Artículo 13: El Gobierno de México hace reserva de este Artículo, visto el texto actual del Artículo 33 de la Constitución Política de los Estados Unidos Mexicanos ${ }^{30}$.

Artículo 25. Inciso b). El Gobierno de México hace igualmente reserva de esta disposición, en virtud de que el Artículo 190 de la Constitución Política de los Estados Unidos Mexicanos dispone que los ministros de los cultos no tendrán voto activo, ni pasivo, ni derecho para asociarse con fines políticos"31.

Estas reservas fueron formuladas por México en el momento de su adhesión al Pacto internacional de derechos civiles y políticos en el año 1981, de manera que no es de extrañar que las mismas hayan sufrido una retirada parcial ${ }^{32}$.

En este caso, el 15 de marzo de 2002, el Gobierno de México notificó al Secretario General la primera retirada parcial de su reserva al artículo 25 (b) formulada en el momento de la adhesión. De igual modo, el 11 de julio de 2014 el Secretario General recibió por parte de México la retirada de su reserva relativa al artículo 13 del Pacto. De tal manera que, en la actualidad, la reserva figura depositada ante el Secretario General con la siguiente redacción:

"Article 25, subparagraph b): The Government of Mexico also makes a reservation to this provision, since article 130 of the Political Constitution of the United Mexican States provides that ministers of religion shall have neither a passive vote nor the right to form associations for political purposes" 33 .

Por consiguiente, la reserva pasa a tener un menor alcance respecto de la modificación del artículo 25 (b) del Pacto y a reconocer a los ministros de religión el derecho al voto activo que antes era objeto de reserva.

Por otro lado, en cuanto a las declaraciones interpretativas, hemos de tener en cuenta que pueden constituir verdaderas reservas en determinadas ocasiones como señalábamos

${ }^{30}$ El artículo 33 de la Constitución Política de los Estados Mexicanos establece que: "Son personas extranjeras las que no posean las calidades determinadas en el artículo 30 constitucional y gozarán de los derechos humanos y garantías que reconoce esta Constitución.

El Ejecutivo de la Unión, previa audiencia, podrá expulsar del territorio nacional a personas extranjeras con fundamento en la ley, la cual regulará el procedimiento administrativo, así como el lugar y tiempo que dure la detención.

Los extranjeros no podrán de ninguna manera inmiscuirse en los asuntos políticos del país". Vid. Constitución Política de los Estados Mexicanos, publicado en el D.O.F. el 5 de febrero de 1917, texto vigente última reforma publicada en D.O.F. 27-08-18.

${ }^{31}$ Reserva disponible en la adhesión de México al Pacto Internacional de Derechos Civiles y Políticos, publicado en Diario Oficial de la Federación (DOF), Decreto Promulgatorio DO 20 de mayo de 1981.

No obstante, el artículo de la Constitución mexicana que figura en el texto de la reserva publicada en el vínculo web https://treaties.un.org/Pages $/$ ViewDetails.aspx?src $=$ TREATY\&mtdsg_no $=I V$ $4 \&$ chapter $=4 \&$ clang $=$ en, de Naciones Unidas, es el 130. En este sentido, los apartados d) y e) del artículo 130 de la Constitución establecen que los ministros de cultos no podrán desempeñar cargos públicos. Como ciudadanos tendrán derecho a votar, pero no a ser votados. Asimismo, dispone, entre otras cosas, que los ministros no podrán asociarse con fines políticos ni realizar proselitismo a favor o en contra de candidato, partido o asociación política alguna.

${ }^{32}$ Vid. Decreto Promulgatorio DOF 16 de enero de 2002, de retiro parcial de la reserva que el Gobierno de México formuló al artículo 25 b) del Pacto internacional de derechos civiles y políticos. Puesto que "Tanto la reserva como la objeción pueden ser retiradas en cualquier momento, salvo que el tratado disponga otra cosa", vid., SOLARI YRIGOYEN, H. "Las reservas a los Tratados Internacionales de Derechos Humanos", Agenda Internacional, $\mathrm{n}^{\circ} 8$ : pp. 72-85, p. 75.

$33 \quad$ Vid. https://treaties.un.org/Pages/ViewDetails.aspx?src=TREATY\&mtdsg_no=IV$4 \&$ chapter $=4 \&$ clang $=\_$en $\# 33$. 
ut supra, muchas de las cuales podrían considerarse inválidas. De ahí que también deban ser objeto de estudio las declaraciones interpretativas formuladas por este Estado.

En este sentido, el Gobierno de México ha formulado ciertas declaraciones interpretativas al Pacto internacional de derechos civiles y políticos, concretamente a los artículos $9.3^{34}$ y $18^{35}$ :

“Artículo 9, párrafo 3. De acuerdo con la Constitución Política de los Estados Unidos Mexicanos y sus leyes reglamentarias, todo individuo goza de las garantías que en materia penal se consagran, y en consecuencia, ninguna persona podrá ser ilegalmente detenida o presa. Sin embargo, si por falsedad en la denuncia o querella, cualquier individuo sufre un menoscabo en este derecho esencial, tiene entre otras cosas, según lo dispongan las propias leyes, la facultad de obtener una reparación efectiva y justa.

Artículo 18. De acuerdo con la Constitución Política de los Estados Unidos Mexicanos todo hombre es libre de profesar la creencia religiosa que más le agrade y para practicar las ceremonias, devociones o actos del culto respectivo, con la limitación, respecto de los actos religiosos de culto público de que deberán celebrarse precisamente en los templos y, respecto de la enseñanza, de que no se reconoce validez oficial a los estudios hechos en los establecimientos destinados a la enseñanza profesional de los ministros de los cultos. El Gobierno de México considera que estas limitaciones están comprometidas dentro de las que establece el párrafo 3 de este Artículo" 36 .

En el caso de estas declaraciones interpretativas, México hace alusión a su Derecho interno sin determinar a qué preceptos constitucionales se refiere, lo que podría obstaculizar conocer el alcance de dicha declaración y, por consiguiente, la asunción de obligaciones convencionales por parte de este Estado ${ }^{37}$.

Asimismo, formula una declaración interpretativa a la Convención internacional sobre la protección de los derechos de todos los trabajadores migratorios y de sus familiares. De hecho, consideramos que esta última podría constituir en realidad una reserva contraria al objeto y fin de la Convención, ya que en la misma establece, entre

\footnotetext{
${ }^{34}$ Disponible en la adhesión de México al Pacto Internacional de Derechos Civiles y Políticos, publicado en Diario Oficial de la Federación (DOF), Decreto Promulgatorio DO 20 de mayo de 1981. Aunque en el enlace web de Naciones Unidas, esta declaración interpretativa aparece formulada al párrafo 5 de este artículo 9. Vid., https://treaties.un.org/Pages/ViewDetails.aspx?src=TREATY\&mtdsg_no=IV$4 \&$ chapter $=4 \&$ clang $=$ en. El apartado 5 del artículo 9 dice: "Toda persona que haya sido ilegalmente detenida o presa, tendrá el derecho efectivo a obtener reparación".

35 Relativo a la libertad de pensamiento, conciencia y religión. El párrafo 3 al que hace referencia la declaración interpretativa dispone que "la libertad de manifestar la propia religión o las propias creencias estará sujeta únicamente a las limitaciones prescritas por la ley que sean necesarias para proteger la seguridad, el orden, la salud o la moral públicos, o los derechos y libertades fundamentales de los demás". Diario Oficial de la Federación (DOF), Decreto Promulgatorio DO 20 de mayo de 1981.

${ }^{36}$ Vid. La adhesión de México al Pacto Internacional de Derechos Civiles y Políticos, publicado en Diario Oficial de la Federación (DOF), Decreto Promulgatorio DO 20 de mayo de 1981.

${ }^{37}$ Máxime si tenemos en cuenta el Comentario General no 24, de 11 de noviembre de 1994, aprobado por el Comité de Derechos Humanos, sobre cuestiones relacionadas con las reservas formuladas con ocasión de la ratificación del Pacto o de sus Protocolos Facultativos, o de la adhesión a ellos, o en relación con las declaraciones hechas de conformidad con el artículo 41 del Pacto. Concretamente, el párrafo 12 de este Comentario General hace referencia a aquellas reservas fundamentadas en la legislación nacional, como es el caso de las declaraciones de México, y especialmente aquellas formuladas en términos generales.
} 
otras cosas, que: “(...) todas las disposiciones de esta Convención se aplicarán de conformidad con su legislación nacional" ${ }^{38}$.

A tenor de esta observación por parte de México, podemos plantearnos la posibilidad de que un ulterior incumplimiento de alguna disposición convencional por parte de este Estado pudiera ser justificado por parte del Gobierno mexicano invocando que existe disconformidad de la misma con su derecho interno, lo que sería contrario a lo establecido en el artículo 27 CVDT.

Otra de las declaraciones interpretativas de México es la formulada a la Convención sobre la Imprescriptibilidad de los Crímenes de Guerra y de los Crímenes de Lesa Humanidad, cuyo tenor literal dispone:

"Con fundamento en el artículo 14 de la Constitución Política de los Estados Unidos Mexicanos, el Gobierno de México, al ratificar la Convención sobre la Imprescriptibilidad de los Crímenes de Guerra y de los Crímenes de Lesa Humanidad, adoptada por la Asamblea General de las Naciones Unidas el 26 de noviembre de 1968, entenderá que únicamente considerará imprescriptibles los crímenes que consagra la Convención, cometidos con posterioridad a su entrada en vigor para México"39.

Al respecto, el artículo 14 de la Constitución dispone que "a ninguna ley se dará efecto retroactivo en perjuicio de persona alguna", a pesar de que el primer artículo de esta Convención establece que los crímenes de guerra y de lesa humanidad son imprescriptibles, cualquiera que sea la fecha en que se hayan cometido. De esta manera, cabría preguntarse si la declaración interpretativa es una reserva y afecta a un elemento esencial del convenio y, por tanto, podría tener la consideración de reserva contraria al objeto y fin del tratado en los términos y con las consecuencias previstas en la CVDT.

Igualmente, México formula una declaración interpretativa al adherirse al Pacto internacional de Derechos económicos, sociales y culturales, en virtud de la cual, "el Gobierno de México lo hace en el entendimiento de que el Artículo 8 del aludido Pacto ${ }^{40}$ se aplicará en la República Mexicana dentro de las modalidades y conforme a los procedimientos previstos en las disposiciones aplicables de la Constitución Política de los Estados Unidos Mexicanos y de sus leyes reglamentarias"41.

Al igual que ocurría con la declaración formulada al Pacto internacional de derechos civiles y políticos enunciada con anterioridad, no detalla las disposiciones aplicables de la Constitución, lo que impide determinar el alcance de la reserva y, por consiguiente, su compatibilidad con el objeto y fin del tratado.

Sin embargo, a pesar de todo lo expuesto, ninguna de las reservas o declaraciones interpretativas formuladas por México que han sido analizadas ha recibido objeciones por parte de ningún Estado, lo que representa un indicio de que los Estados Partes han

\footnotetext{
${ }^{38}$ Declaración en la Convención Internacional sobre la protección de los derechos de todos los trabajadores migratorios y de sus familiares, hecho en Nueva York el 18 de diciembre de 1990, ratificación por México de 8 de marzo de 1999, Decreto Promulgatorio DOF, 13 de agosto de 1999. Igualmente disponible en: https://treaties.un.org/Pages/ViewDetails.aspx?src $=$ TREATY\&mtdsg_no $=I V$ -

$13 \&$ chapter $=4 \&$ clang $=$ en.

39 Vid. Convención sobre la Imprescriptibilidad de los Crímenes de Guerra y de los Crímenes de Lesa Humanidad, publicado en DOF, de 22 de abril de 2002.

40 Relativo al derecho a la libertad sindical: formar sindicatos y afiliarse al de su elección, y al funcionamiento de los mismos.

41 Vid. Instrumento de adhesión de 23 de marzo de 1981, publicado en Diario Oficial de la Federación (DOF), Decreto Promulgatorio DO, de 12 de mayo de 1981.
} 
considerado válidas las declaraciones formuladas en relación con el contenido de estos convenios internacionales de Derechos Humanos.

Por otro lado, resulta interesante subrayar el dato relativo a que México ha retirado varias reservas y declaraciones interpretativas, algunas de las cuales podrían poner en entredicho las obligaciones convencionales asumidas por este Estado en relación con la disposición del tratado a la que hace referencia, lo que también supone una mayor asunción de obligaciones internacionales sobre Derechos Humanos por parte del Estado mexicano. Entre ellas, encontramos la declaración interpretativa que México formuló a la Convención sobre los derechos de las personas con discapacidad, en la que decía que, en caso de conflicto entre su normativa interna y el párrafo 2 del artículo 12 del tratado ${ }^{42}$, se aplicará la norma que confiera mayor protección legal. El precepto al que se refiere la declaración interpretativa podría tener, a nuestro juicio, consideración de elemento esencial de la Convención, pero además, hace referencia a su legislación nacional, lo cual contraviene lo establecido en la CVDT.

Sin embargo, la misma fue retirada ${ }^{43}$, lo que puede resultar indicativo del reconocimiento de su incompatibilidad por parte de este Estado, así como un avance hacia la promoción de los Derechos contenidos en este instrumento convencional.

Igualmente México retiró su reserva a la Convención internacional sobre la protección de los derechos de todos los trabajadores migratorios y de sus familiares, formulada respecto del "párrafo 4 del Artículo 22 de esta Convención, exclusivamente por lo que se refiere a la aplicación del artículo 33 de la Constitución Política de los Estados Unidos Mexicanos ${ }^{44}$ y 125 de la Ley General de Población ${ }^{45}$ ". 46 Es decir, justificada con base en su Derecho nacional.

Hemos de decir al respecto que el artículo 22.4 del Convenio ${ }^{47}$ se refiere a los derechos de los individuos para oponerse a su expulsión, lo que podría poner en cuestión la validez de dicha reserva. No obstante, la misma fue retirada, lo cual merece una valoración positiva a la luz de la posible incompatibilidad de esa reserva con el objeto y fin del tratado.

\footnotetext{
${ }^{42}$ Que establece que "los Estados Partes reconocerán que las personas con discapacidad tienen capacidad jurídica en igualdad de condiciones con las demás en todos los aspectos de la vida". Vid. Convención sobre los derechos de las personas con discapacidad y protocolo facultativo, DOF 2 de mayo de 2008.

${ }^{43}$ Retirada de la reserva disponible en el Decreto publicado en el Diario Oficial, de jueves 8 de diciembre de 2011.

${ }^{44}$ Que establece la condición de extranjero y además que "el Ejecutivo de la Unión, previa audiencia, podrá expulsar del territorio nacional a personas extranjeras con fundamento en la ley, la cual regulará el procedimiento administrativo, así como el lugar y tiempo que dure la detención". Y que "Los extranjeros no podrán de ninguna manera inmiscuirse en los asuntos políticos del país." Vid. D.O.F. el 5 de febrero de 1917, texto vigente, última reforma publicada en D.O.F. 27 de agosto de 2018.

${ }^{45}$ Que establece los supuestos en los que se cancelará la calidad migratoria y la persona será expulsada del país. Artículo que en la actualidad se encuentra derogado. Vid., última reforma publicada DOF 12-07-18. Texto de la disposición disponible en el Decreto publicado en DOF 7 de enero de 1974.

46 Retirada de la reserva accesible en: https://treaties.un.org/Pages/ViewDetails.aspx?src $=$ TREATY\&mtdsg_no $=I V$ $13 \&$ chapter $=4 \&$ clang $=\_$en.

${ }^{47}$ Cuyo tenor literal establece que "Salvo cuando una autoridad judicial dicte una decisión definitiva, los interesados tendrán derecho a exponer las razones que les asistan para oponerse a su expulsión, así como a someter su caso a revisión ante la autoridad competente, a menos que razones imperiosas de seguridad nacional se opongan a ello. Hasta tanto se haga dicha revisión, tendrán derecho a solicitar que se suspenda la ejecución de la decisión de expulsión", vid. DOF de 13 de agosto de 1999.
} 


\subsection{Objeciones.}

Por otro lado, como ya hemos puesto de manifiesto, debemos someter a los criterios de validez no sólo las reservas y las declaraciones interpretativas formuladas por México, sino que resulta igualmente interesante evaluar la práctica de México en relación con la formulación de objeciones a las reservas y declaraciones interpretativas de otros Estados, en especial respecto de aquellas declaraciones cuyo contenido puede constituir una reserva que ponga en peligro la integridad y los objetivos fundamentales del tratado ${ }^{48}$.

En este aspecto, resulta significativo el ejemplo de la declaración formulada por la República Bolivariana de Venezuela a la Convención Internacional sobre la protección de los Derechos de todos los trabajadores migratorios y de sus familiares ${ }^{49}$, que cuenta una única objeción, concretamente por parte de México.

Como se desprende de su tenor literal, dicha declaración interpretativa tiene por objeto que el derecho a afiliarse a un sindicato se aplique en exclusiva a los trabajadores migrantes y no a sus familiares ${ }^{50}$, a pesar de que el artículo 26 de la Convención establece que este derecho se hará extensivo a los familiares de estos trabajadores ${ }^{51}$. En este contexto, la objeción de México se basa en que esta declaración por parte de Venezuela constituye en realidad una reserva y que la misma es contraria al objeto y fin de la convención ${ }^{52}$. A la luz de estos datos, consideramos que la afirmación contenida en la

\footnotetext{
${ }^{48}$ De acuerdo con GARCÍA RICO, la formulación de objeciones representa una postura más firme respecto de este tipo de reservas, lo que constituye un avance en la búsqueda de soluciones al problema de las reservas a tratados que tienen por objeto la protección de intereses generales de la sociedad internacional. Vid. GARCÍA RICO, E.M. (2010), "Algunas reflexiones en torno a la práctica española sobre reservas a los tratados de Derechos Humanos de ámbito universal”, Colección Escuela Diplomática, n¹6, vol. 2: pp. 235-247, p. 237.

${ }^{49}$ El texto de la declaración interpretativa establece:

"With regard to the provisions of article 26, paragraph 1, of the International Convention on the Protection of the Rights of All Migrant Workers and Members of their Families, the Bolivarian Republic of Venezuela considers that the right to join freely any trade union, contained in subparagraph (b), applies exclusively to migrant workers.

Considering the provisions of article 92, paragraph 2, of the International Convention on the Protection of the Rights of All Migrant Workers and Members of their Families, the Bolivarian Republic of Venezuela declares that it does not consider itself bound by paragraph 1 of said article. Consequently, it does not consider itself bound to resort to arbitration as a means of dispute settlement, and does not recognize the binding jurisdiction of the International Court of Justice".

Disponible en https://treaties.un.org/Pages/ViewDetails.aspx?src=TREATY\&mtdsg_no=IV$13 \&$ chapter $=4 \&$ clang $=$ en\#EndDec.

${ }^{50}$ Por su parte, el segundo párrafo de esta declaración se encuentra expresamente contemplada y permitida en el párrafo 2 del artículo 92, que da la opción de que el Estado parte declare que no se considera obligado por el párrafo 1 .

${ }^{51}$ En concreto, este artículo dice que “1. Los Estados Partes reconocerán el derecho de los trabajadores migratorios y sus familiares a:

a) Participar en las reuniones y actividades de los sindicatos o de cualesquiera otras asociaciones establecidas conforme a la ley, con miras a proteger sus intereses económicos, sociales, culturales y de otra índole, con sujeción solamente a las normas de la organización pertinente;

b) Afiliarse libremente a cualquier sindicato o a cualquiera de las asociaciones citadas, con sujeción solamente a las normas de la organización pertinente;

c) Solicitar ayuda y asistencia de cualquier sindicato o de cualquiera de las asociaciones citadas.

2. El ejercicio de tales derechos sólo podrá estar sujeto a las restricciones previstas por la ley que sean necesarias en una sociedad democrática en interés de la seguridad nacional o el orden público o para proteger los derechos y libertades de los demás." Vid.
} https://www.ohchr.org/SP/ProfessionalInterest/Pages/CMW.aspx.

52 With regard to the declaration made by Venezuela upon ratification: After analyzing the declaration made by the Bolivarian Republic of Venezuela regarding the International 
objeción formulada por México resulta acertada en el sentido de considerar que esta declaración interpretativa contiene en realidad una reserva, ya que la declaración de Venezuela tiene por objeto excluir o modificar los efectos jurídicos de la disposición y no aclarar su sentido. Sin embargo, resulta llamativo sobre el particular el hecho de que ningún otro Estado haya formulado una objeción similar respecto de esta declaración interpretativa ${ }^{53}$.

En términos parecidos se pronuncia México en su objeción a la reserva formulada por Irán a la Convención sobre los derechos de las personas con discapacidad ${ }^{54}$, una vez más bajo la denominación de declaración interpretativa. En este sentido, la reserva formulada por Irán a la que nos referimos, establece que:

"... with regard to Article 46, the Islamic Republic of Iran declares that it does not consider itself bound by any provisions of the Convention, which may be incompatible with its applicable rules." 55

Según el artículo 46 de la Convención, al que esta declaración hace referencia, "no se permitirán reservas incompatibles con el objeto y propósito de la presente Convención" 56 .

Convention on the Protection of the Rights of All Migrant Workers and Members of Their Families, the Government of the United Mexican States has reached the conclusion that the declaration in fact constitutes a reservation.

This reservation, which is designed to exclude the legal effects of article 26, paragraph 1, of the Convention, is contrary to the object and purpose of the Convention and, particularly, to the right of members of the families of migrant workers to join freely any trade union or association, and therefore contravenes the provisions of article 91, paragraph 2, of the Convention and article 19 of the Vienna Convention on the Law of Treaties.

This objection does not prevent the entry into force of the Convention between the Bolivarian Republic of Venezuela and the United Mexican States. Consequently, the Convention will apply between the two States without the Bolivarian Republic of Venezuela being able to avail itself of the above-mentioned reservation. Esta objeción por parte de México está disponible en: https://treaties.un.org/Pages/ViewDetails.aspx?src=TREATY\&mtdsg_no $=I V$ -

$13 \&$ chapter $=4 \&$ clang $=$ en\#EndDec .

${ }^{53}$ Puesto que no es extraño que en aquellos casos en los que una declaración interpretativa constituye una reserva de forma clara, la misma reciba numerosas objeciones por parte de distintos Estados.

${ }^{54}$ La objeción dice que:

"With regard to the reservation made by the Islamic Republic of Iran upon accession:

Having examined the declaration made by the Islamic Republic of Iran with respect to the Convention, the United Mexican States has concluded that the declaration is, in fact, a reservation. This reservation, which aims to exclude the legal effects of certain provisions of the Convention, is incompatible with the object and purpose of that instrument. Indeed, the declaration is worded in such a way that it could hinder the realization of normative provisions of the Convention, including those of articles 4 and 1 , and thus is in breach of article 46 of the Convention and article 19 of the Vienna Convention on the Law of Treaties. It should be noted that article 27 of the Vienna Convention codified the principle of international law whereby a party may not invoke the provisions of its domestic law as justification for its failure to comply with a treaty. The claim that domestic laws take precedence over the provisions of treaties that are in force for the Parties is therefore inadmissible.

This objection shall not preclude the entry into force of the Convention between the Islamic Republic of Iran and the United Mexican States."

Disponible en https://treaties.un.org/Pages/ViewDetails.aspx?src=TREATY\&mtdsg_no=IV$15 \&$ chapter $=4 \&$ clang $=$ en $\#$ EndDec.

Igualmente, el Gobierno de los Estados Unidos Mexicanos establece que la declaración/reserva de la República de Surinam es realmente una reserva con declaraciones contrarias al objeto y fin de la convención.

55 Texto accesible en: https://treaties.un.org/pages/ViewDetails.aspx?src=TREATY\&mtdsg_no=IV$15 \&$ chapter $=4 \&$ clang $=$ en $\#$ EndDec .

${ }^{56} \mathrm{Al}$ igual que establece el artículo 19 del Convenio de Viena sobre el Derecho de los Tratados. 
A tenor de lo cual, no resulta extraño que esta declaración de Irán, cuyo objeto es excluir los efectos jurídicos de aquellas disposiciones del convenio incompatibles con su derecho interno, sea calificada por parte de México y de otros muchos Estados como una reserva incompatible con el objeto y fin del tratado, puesto que la misma podría obstaculizar la ejecución de ciertas disposiciones normativas de la convención. Se trataría, por tanto, de una reserva contraria al artículo 27 del CVDT, en virtud del cual una parte no podrá invocar disposiciones de su derecho interno para justificar el incumplimiento de un tratado. Por todo ello, y tomando igualmente en consideración los artículos 46 de la Convención sobre los derechos de las personas con discapacidad y 19 CVDT, la formulación de este tipo de reservas no estaría permitida.

Como se ha puesto de manifiesto con anterioridad, no se consideran válidas aquellas reservas que son contrarias al objeto y fin de la convención: ya sea por tratarse de una reserva con carácter general, por supeditar la aplicación de la totalidad del texto del tratado a la ley nacional, o bien por tratarse de reservas que se refieren a elementos esenciales del tratado. En este sentido, resultan muy ilustrativas determinadas reservas formuladas a la Convención sobre la eliminación de todas las formas de discriminación contra la mujer ${ }^{57}$, como la formulada por la República de Mauricio con respecto a los apartados b) y d) del párrafo 1 del artículo $11^{58}$ y el apartado g) del párrafo 1 del artículo $16^{59}$.

En este caso, a pesar de que la reserva hace referencia expresa sólo a dos artículos de la convención, en concreto el 11 y el 16, esto no impide a México formular la siguiente objeción:

"The Government of the United Mexican States has studied the content of the reservations made by Mauritius to article 11, paragraph 1 (b) and (d), and article 16, paragraph 1 (g), of the Convention and has concluded that they should be considered invalid in the light of article 28, paragraph 2, of the Convention, because they are incompatible with its object and purpose.

Indeed, these reservations, if implemented, would inevitably result in discrimination against women on the basis of sex, which is contrary to all the articles of the Convention. The principles of equal rights of men and women and nondiscrimination on the basis of sex, which are embodied in the second preambular paragraph and Article 1, paragraph 3, of the Charter of the United Nations, to which Mauritius is a signatory, and in articles 2 and 16 of the Universal Declaration of

\footnotetext{
${ }^{57}$ Instrumento de ratificación de 23 de marzo de 1981, publicado en Diario Oficial de la Federación (DOF), Decreto Promulgatorio DO, de 12 de mayo de 1981.

${ }^{58}$ Que dicen que:

"Los Estados Partes adoptarán todas las medidas apropiadas para eliminar la discriminación contra la mujer en la esfera del empleo a fin de asegurar condiciones de igualdad entre hombres y mujeres, mismos derechos, en particular:

b) El derecho de las mismas oportunidades de empleo, inclusive a la aplicación de los mismos criterios de selección en cuestiones de empleo.

d) El derecho a igual remuneración, inclusive prestaciones, y a igualdad de trato con respecto a un trabajo de igual valor, así como a igualdad de trato con respecto a la evaluación de la calidad del trabajo." Diario Oficial de la Federación (DOF), Decreto Promulgatorio DO, de 12 de mayo de 1981.

${ }^{59}$ Que dice que:

"Los Estados Partes adoptarán todas las medidas adecuadas para eliminar la discriminación contra la mujer en todos los asuntos relacionados con el matrimonio y las relaciones familiares y, en particular, asegurarán, en condiciones de igualdad entre hombres y mujeres:

g) Los mismos derechos personales como marido y mujer, entre ellos el derecho a elegir apellido, profesión y ocupación.” Diario Oficial de la Federación (DOF), Decreto Promulgatorio DO, de 12 de mayo de 1981.
} 
Human Rights of 1948, were previously accepted by the Government of Mauritius when it acceded, on 12 December 1973, to the International Covenant on Civil and Political Rights and the International Covenant on Economic, Social and Cultural Rights. The above principles were stated in article 2, paragraph 1, and article 3 of the former Covenant and in article 2, paragraph 2, and article 3 of the latter. Consequently, it is inconsistent with these contractual obligations previously assumed by Mauritius for its Government now to claim that it has reservations, on the same subject, about the 1979 Convention.

The objection of the Government of the United Mexican States to the reservations in question should not be interpreted as an impediment to the entry into force of the 1979 Convention between the United Mexican States and Mauritius"60.

Compartimos la posición de México al considerar que se trata de una reserva incompatible con el objeto de este tratado internacional, pues debemos tener en cuenta que estos artículos se refieren a la igualdad de empleo y remuneración entre hombres y mujeres y a los mismos derechos como marido y mujer en la elección del apellido, profesión y ocupación, de manera que la aceptación de esta reserva supondría una discriminación por razón de género, contraria al propósito de este tratado internacional. De hecho, esta reserva recibió un gran número de objeciones, lo que llevó a que fuese finalmente retirada por el Estado que la había formulado ${ }^{61}$ : una práctica cada vez más frecuente, aunque poco habitual en el pasado ${ }^{62}$.

En la misma línea, México formuló una objeción a una reserva formulada por la República de Turquía por considerarla contraria al objeto y fin de la Convención por razones similares, que fue igualmente retirada por parte de este Estado. Dicha reserva se refería a los párrafos 2 y 4 del artículo $15^{63}$ y a los párrafos $\left.1 \mathrm{c}\right)$, d), f) ${ }^{64}$ y g) del artículo 16 de la Convención.

El gobierno de México ha formulado otras objeciones, mutatis mutandis pero idénticas en esencia, a numerosas reservas formuladas por otros Estados a la Convención

\footnotetext{
$60 \quad$ Vid, $\quad$ https://treaties.un.org/Pages/ViewDetails.aspx?src $=$ TREATY\&mtdsg_no=IV$8 \&$ chapter $=4 \&$ clang $=$ en $\# 82$.

61 Vid. Nota 41 en https://treaties.un.org/Pages/ViewDetails.aspx?src=TREATY\&mtdsg_no=IV$8 \&$ chapter $=4 \&$ clang $=$ en $\# 41$.

62 Vid., por todos, HORN, F. (1988), Reservations and Interpretative..., op. cit., p. 226.

${ }^{63}$ Cuyo texto dice:

15.2. "Los Estados Partes reconocerán a la mujer, en materias civiles, una capacidad jurídica idéntica a la del hombre y las mismas oportunidades para el ejercicio de esa capacidad. En particular, le reconocerán a la mujer iguales derechos para firmar contratos y administrar bienes y le dispensarán un trato igual en todas las etapas del procedimiento en las cortes de justicia y los Tribunales."

15.4. "Los Estados Partes reconocerán al hombre y a la mujer los mismos derechos con respecto a la legislación relativa al derecho de las personas a circular libremente y a la libertad para elegir su residencia y domicilio”. Diario Oficial de la Federación (DOF), Decreto Promulgatorio DO, de 12 de mayo de 1981.

${ }^{64}$ Que dice, en relación con el matrimonio y las relaciones familiares, que:

“c) Los mismos derechos y responsabilidades durante el matrimonio y con ocasión de su disolución.

d) Los mismos derechos y responsabilidades como progenitores, cualquiera que sea su estado civil, en materias relacionadas con sus hijos; en todos los casos, los intereses de los hijos serán la consideración primordial.

f) Los mismos derechos y responsabilidades respecto de la tutela, curatela, custodia y adopción de los hijos, o instituciones análogas cuando quiera que estos conceptos existan en la legislación nacional; en todos los casos, los intereses de los hijos serán la consideración primordial." Vid. Diario Oficial de la Federación (DOF), Decreto Promulgatorio DO, de 12 de mayo de 1981.
} 
sobre la eliminación de todas las formas de discriminación contra la mujer ${ }^{65}$. Todas ellas referidas a artículos que contienen derechos esenciales de la misma, como ya se ha puesto de manifiesto.

En otro orden de consideraciones, México objetó la reserva formulada por Estados Unidos al artículo IX del Convenio para la prevención y la sanción del delito de genocidio $^{66}$, relativo a la jurisdicción de la Corte Internacional de Justicia ${ }^{67}$.

La objeción formulada por México a esta reserva establece que:

"The Government of Mexico believes that the reservation made by the United States Government to article IX of the aforesaid Convention should be considered invalid because it is not in keeping with the object and purpose of the Convention, nor with the principle governing the interpretation of treaties whereby no State can invoke provisions of its domestic law as a reason for not complying with a treaty.

If the aforementioned reservation were applied, it would give rise to a situation of uncertainty as to the scope of the obligations which the United States Government would assume with respect to the Convention.

Mexico's objection to the reservation in question should not be interpreted as preventing the entry into force of the 1948 Convention between the [Mexican] Government and the United States Government" ${ }^{\prime \prime}$.

Por tanto, la objeción de México se fundamenta en que dicha reserva no debería considerarse válida porque no está en consonancia con el objeto y propósito de la Convención. Asimismo, establece que la misma es contraria al principio en virtud del cual ningún Estado puede invocar disposiciones de su legislación interna como motivo para no cumplir las disposiciones convencionales y declara que esta reserva da lugar a una situación de incertidumbre, puesto que se trata de una reserva general que no permite conocer el alcance de las obligaciones que el Gobierno de Estados Unidos asumiría con respecto a esta Convención.

En este sentido, compartimos el criterio de México, aunque entendemos que esta objeción debería contemplar en exclusiva el segundo párrafo de la reserva formulada por Estados Unidos, concerniente a que ninguna disposición de la Convención puede exigir o autorizar acciones prohibidas por su Constitución, puesto que una reserva por parte de

\footnotetext{
${ }^{65}$ En concreto a las reservas formuladas por Bangladesh, Jamaica, Nueva Zelanda, la República de Corea, la República de Chipre, la República Árabe de Egipto, Reino de Tailandia, Irak y Libia. Vid. https://treaties.un.org/Pages/ViewDetails.aspx?src=TREATY\&mtdsg_no=IV-8\&chapter=4\&clang=_en.

${ }^{66}$ Este artículo IX del Convenio para la prevención y la sanción del delito de genocidio señala que: Las controversias entre las Partes contratantes, relativas a la interpretación, aplicación o ejecución de la presente Convención, incluso las relativas a la responsabilidad de un Estado en materia de genocidio o en materia de cualquiera de los otros actos enumerados en el artículo III, serán sometidas a la Corte Internacional de Justicia a petición de una de las Partes en la controversia. Vid. DOF 11 de octubre de 1952.

${ }^{67}$ El tenor literal de la reserva establece:

"1. That with reference to article IX of the Convention, before any dispute to which the United States is a party may be submitted to the jurisdiction of the International Court of Justice under this article, the specific consent of the United States is required in each case.

2. That nothing in the Convention requires or authorizes legislation or other action by the United States of America prohibited by the Constitution of the United States as interpreted by the United States." Texto de la reserva disponible en: https://treaties.un.org/Pages/ViewDetails.aspx?src=TREATY\&mtdsg_no=IV$1 \&$ chapter $=4 \&$ clang $=$ en $\#$ EndDec.

68 Disponible en https://treaties.un.org/Pages/ViewDetails.aspx?src=TREATY\&mtdsg_no=IV$1 \&$ chapter $=4 \&$ clang $=$ en $\#$ EndDec.
} 
los Gobiernos a someterse a la jurisdicción de la Corte Internacional de Justicia responde a la práctica internacional de los Estados de forma habitual ${ }^{69}$, y no consideramos que la misma sea contraria al objeto y fin del tratado.

Pero estas no son las únicas objeciones que, sobre la base de la incompatibilidad de la reserva con el objeto y fin del tratado, realiza México. Así ocurre con la objeción a la reserva formulada por la República de Yemen a los apartados c y d iv), vi) y vii) del artículo 5 de la Convención internacional sobre la eliminación de todas las formas de discriminación racial ${ }^{70}$, relativos al reconocimiento, sin distinción por razón de raza, color $\mathrm{u}$ origen, de derechos políticos -en concreto, el sufragio activo y pasivo- y de derechos civiles -al matrimonio y a la elección del cónyuge, el derecho a heredar y el derecho a la libertad de pensamiento, conciencia y religión- ${ }^{71}$. Objeta también la reserva formulada por el Reino de Baréin al Pacto internacional de derechos civiles y políticos, por suponer la sujeción de las disposiciones convencionales a la Sharia islámica. Sin embargo, en este último caso, la reserva formulada por parte de Baréin no llegó a ser aceptada, debido al gran número de objeciones recibidas ${ }^{72}$.

A la luz de estos datos, observamos que México tiene una práctica tendente a la formulación de objeciones a aquellas reservas que considera incompatibles con el objeto y fin del tratado. Asimismo, por lo que respecta a la práctica de México en materia de reservas y declaraciones interpretativas, como hemos tenido ocasión de comprobar, a pesar de la abundante ratificación de convenios sobre Derechos Humanos este Estado no destaca por la formulación de reservas y declaraciones interpretativas, lo que representa un elemento acorde con la debida protección de los Derechos Humanos al aceptar estos instrumentos convencionales en su integridad.

\footnotetext{
69 Tanto es así, que en el presente Convenio encontramos, en la fecha de redacción de la presente publicación, un total de 16 reservas al artículo IX. En muchos casos una reserva de este tipo acabó siendo retirada.

${ }^{70}$ La objeción dice:

"With regard to reservation made by Yemen concerning article 5 (c) and article 5 (d) (iv), (vi) and (vii): The Government of the United Mexican States has concluded that, in view of article 20 of the Convention, the reservation must be deemed invalid, as it is incompatible with the object and purpose of the Convention. Said reservation, if implemented would result in discrimination to the detriment of a certain sector of the population and, at the same time, would violate the rights established in articles 2, 16 and 18 of the Universal Declaration of Human Rights of 1948. The objection of the United Mexican States to the reservation in question should not be interpreted as an impediment to the entry into force of the Convention of 1966 between the United States of Mexico and the Government of Yemen." Vid. https://treaties.un.org/Pages/ViewDetails.aspx?src $=$ TREATY\&mtdsg_no $=I V$ $2 \&$ chapter $=4 \&$ clang $=$ en $\#$ top.

${ }^{71}$ Vid. artículo 5 de la Convención internacional sobre la eliminación de todas las formas de discriminación racial. Instrumento de ratificación de 20 de febrero de 1975, publicado en Diario Oficial de la Federación (DOF), Decreto Promulgatorio DO, de 13 de junio de 1975.

${ }^{72}$ En este caso, y de conformidad con la práctica depositaria seguida en casos similares, el Secretario General propuso recibir la reserva en cuestión para su depósito a falta de objeciones por parte de cualquiera de los Estados contratantes, dentro de un período de 12 meses. En ausencia de tal objeción, la reserva sería aceptada en depósito al vencimiento del período de 12 meses. Debido a las objeciones recibidas, no fue aceptada en depósito. Vid., https://treaties.un.org/Pages/ViewDetails.aspx?src=TREATY\&mtdsg_no=IV$4 \&$ chapter $=4 \&$ clang $=$ en $\# 15$.
} 


\section{Otros elementos para evaluar el compromiso internacional de México respecto de la protección de los Derechos Humanos}

A pesar de la información proporcionada por las figuras jurídicas analizadas en el anterior apartado, hemos de decir que existen otros elementos que permiten llevar a cabo un análisis exhaustivo y alcanzar conclusiones válidas sobre el compromiso internacional de México en materia de Derechos Humanos.

En efecto, la formulación de reservas no es el único factor de utilidad para evaluar la posición de un país respecto de la asunción de obligaciones internacionales para la protección de los Derechos Humanos. Así, resulta muy interesante el análisis de la participación de los Estados en los protocolos facultativos de estos tratados y, en su caso, la formulación de reservas y objeciones a estos instrumentos convencionales.

Los protocolos facultativos tienen por objeto, en la mayoría de los casos, establecer la competencia de un Comité que actúa como órgano de control en la aplicación de estos convenios internacionales sobre Derechos Humanos. De manera que sólo el Estado que acepte la competencia del Comité, mediante su ratificación expresa, se somete a su valoración, pudiendo incluso recibir denuncias de los particulares bajo su jurisdicción por una aplicación deficiente del convenio.

En relación con la naturaleza de estos órganos, debemos partir de la base de que los Comités que tienen competencia para resolver controversias en relación con el cumplimiento por los Estados de sus obligaciones convencionales en materia de Derechos Humanos no son órganos jurisdiccionales internacionales. No obstante, se trata de órganos técnicos especializados con capacidad para llevar a cabo una interpretación autorizada sobre el correcto cumplimiento del convenio al que se refieran ${ }^{73}$.

La ratificación de estos protocolos representa un elemento realmente significativo para alcanzar conclusiones satisfactorias acerca de la intención de los Estados de cumplir sus obligaciones internacionales en materia de Derechos Humanos. Esto es así, pues debemos tener en cuenta que los Comités de los tratados internacionales depositados por Naciones Unidas tienen la facultad de examinar de forma periódica los informes presentados por los Estados Partes y formular recomendaciones. De manera que podemos considerar que el Estado que asume de forma fehaciente el compromiso de velar por la protección de los Derechos contenidos en un tratado, aceptará la competencia del Comité correspondiente y se someterá a su supervisión ${ }^{74}$.

De hecho, hemos de decir que es frecuente que los Estados reciban observaciones de los Comités sobre los informes periódicos presentados. En ellos se relacionan tanto los aspectos positivos como los principales motivos de preocupación y las recomendaciones

\footnotetext{
73 ALIJA FERNÁNDEZ, R.A. y BONET PÉREZ, J. (2016), El control de los Tratados Internacionales de Derechos Humanos: realidad y límites. Una aproximación a la labor de los órganos de expertos desde la perspectiva de la efectividad de los derechos económicos, sociales y culturales. Barcelona: Atelier libros jurídicos, p. 47. Asimismo, los autores se pronuncian acerca del valor jurídico de las decisiones de los órganos de vigilancia de estos convenios internacionales de Derechos Humanos, una cuestión que suscita diversas opiniones doctrinales, pp. 40 y ss. Igualmente, para profundizar sobre este tema vid., por todos, JIMÉNEZ SÁNCHEZ, C. (2019), "Human Rights Committees: Their nature and legal relevance in Spain", Spanish yearbook of international law, vol. 23: pp. 104-128 y CARDONA LLORENS, J. (2019) "The legal value of the views and interim measures adopted by united nations treaty bodies (a response to the opinions of E. Jimenez Pineda, C. Jiménez Sánchez and B. Vázquez Rodríguez)”, Spanish yearbook of international law, vol. 23: pp. 146-163.

${ }^{74}$ Sobre la labor de estos Comités, vid. LÓPEZ HURTADO, C. (2001), ¿Un régimen especial para los tratados de Derechos Humanos dentro del Derecho Internacional? Reservas y denuncias de tratados de Derechos Humanos", Anuario Mexicano de Derecho Internacional, vol. I, pp. 247-289, pp. 253 y ss.
} 
del Comité. Esto supone un margen de mejora para los Estados en su compromiso de aplicación del convenio de Derechos Humanos al que se refiera, de manera que la ratificación de los protocolos facultativos siempre significa un paso hacia la protección de estos Derechos, a pesar de que también suponga recibir críticas negativas por parte del correspondiente Comité.

Pues bien, en cuanto a la ratificación de los protocolos facultativos por parte de México, aunque este país ha aceptado la competencia de varios Comités, ha mostrado una actitud algo menos comprometida que la manifestada con respecto a la ratificación de los tratados. En concreto, ha aceptado los Protocolos Facultativos del Pacto Internacional de Derechos civiles y políticos; de la Convención sobre la eliminación de todas las formas de discriminación contra la mujer; de la Convención contra la tortura y otros tratos o penas crueles, inhumanos o degradantes ${ }^{75}$, así como la competencia del Comité contra la Tortura $^{76}$; el Protocolo Facultativo de la Convención sobre los Derechos del Niño relativo a la Participación de Niños en los Conflictos Armados; el Protocolo Facultativo sobre la venta de niños, la prostitución infantil y la utilización de niños en la pornografía; el Segundo Protocolo Facultativo del Pacto Internacional de Derechos Civiles y Políticos, destinado a abolir la pena de muerte y el Protocolo Facultativo de la Convención sobre los Derechos de las personas con discapacidad.

Por otro lado, en lo que respecta a la ratificación de protocolos facultativos, México ha sido instado por la ONU para ratificar el Protocolo Facultativo del Pacto Internacional de Derechos Económicos, Sociales y Culturales ${ }^{77}$, aunque no lo haya hecho por ahora. No obstante, sí se ha sometido al control de otros muchos Comités, de manera que en esos casos tiene el deber de aportar los informes periódicos que son evaluados por el Comité para que el mismo realice las observaciones oportunas. De esta manera, la promoción de los Derechos Humanos se ve favorecida por la práctica de México en relación con los protocolos facultativos ${ }^{78}$.

Hemos de decir que en materia de reservas la práctica de este Estado resulta bastante uniforme, puesto que tampoco destaca por la formulación de reservas a estos protocolos, incluso al igual que ocurría con las convenciones internacionales, México ha retirado determinadas reservas y declaraciones interpretativas cuyo contenido podría resultar de dudosa validez ${ }^{79}$.

\footnotetext{
${ }^{75}$ Que establece la creación de un Subcomité para la prevención de la tortura y otros tratos o penas crueles, inhumanos o degradantes del Comité contra la tortura.

${ }^{76}$ Creado en virtud del texto de la Convención contra la tortura y otros tratos o penas crueles, inhumanos o degradantes (concretamente en el artículo 17 del texto convencional) y cuya competencia queda reconocida por un Estado en virtud de declaración expresa en ese sentido, de acuerdo con el artículo 22 de la Convención. La declaración de México en este sentido fue formulada el 15 de marzo de 2002, vid. https://treaties.un.org/Pages/ViewDetails.aspx?src $=$ TREATY\&mtdsg_no=IV-9\&chapter=4\&clang=_en. 77 Vid., http://www.un.org/spanish/News/story.asp? NewsID=34016\#.Wi7X2oiCE2w.

${ }^{78} \mathrm{Si}$ bien hemos de apuntar que lo realmente esclarecedor en relación con el compromiso internacional de México es el contenido de las observaciones de estos Comités respecto del cumplimiento efectivo de estos convenios de Derechos Humanos por parte del Estado mexicano, así como las denuncias recibidas contra este país, aunque los mismos no son objeto de estudio en un artículo de estas características.

${ }^{79}$ Así ha ocurrido en el caso del Protocolo Facultativo de la Convención sobre los Derechos del Niño sobre la participación de niños en conflictos armados. En este caso la declaración interpretativa que fue retirada tenía por objeto eximir de responsabilidad al Estado mexicano por el reclutamiento de niños por parte de grupos armados no gubernamentales, lo que entendemos que supone un menoscabo en la protección de las víctimas y que podría ser contraria a un elemento esencial de este protocolo. Vid. el Decreto por el que se aprueba el Retiro de la Declaración Interpretativa formulada por el Gobierno de los Estados Unidos Mexicanos al depositar su instrumento de ratificación del Protocolo Facultativo de la Convención sobre los Derechos del Niño relativo a la Participación de Niños en los Conflictos Armados, adoptado por la
} 


\section{Conclusiones.}

El análisis de la práctica convencional de México en relación con los convenios internacionales sobre Derechos Humanos y sus protocolos facultativos que hemos llevado a cabo pone de manifiesto no sólo la asunción por este país de obligaciones internacionales en esta materia, sino también un examen de la siempre controvertida cuestión de las reservas internacionales a este tipo de tratados.

Al respecto, nuestro estudio nos ha permitido constatar, en primer lugar, que este país ha ratificado y es parte en la actualidad de la totalidad de convenios internacionales de ámbito universal auspiciados por Naciones Unidas para la protección de los Derechos Humanos. En segundo término, que México no suele formular reservas a estos tratados e, incluso, en muchos casos la reserva formulada inicialmente por este Estado acabó siendo retirada.

No obstante, en ocasiones, este país ha formulado determinadas reservas y declaraciones interpretativas cuya validez resulta cuestionada a la luz de los criterios de compatibilidad de las mismas con el objeto y fin del convenio. Sin embargo, dichas reservas no han sido objetadas por otros Estados Partes en esos convenios, lo que podría considerarse como muestra de que no han sido estimadas como tales por los principales sujetos del ordenamiento internacional.

Por otro lado, la práctica de México en materia de objeciones es bastante abundante, así como los casos en que éstas se realizan por considerar que las reservas formuladas por otros Estados Partes en estos convenios son incompatibles con el objeto y fin del tratado $\mathrm{y}$, por tanto, no deberían considerarse válidas. Al hacerlo, este país parece compartir la posición de otros Estados Partes de tal manera que, a pesar de que con carácter general la utilidad de las objeciones a las reservas en aquellos casos en los que la misma "no impide la entrada en vigor del tratado entre el país que formula la reserva y el que formula la objeción" puede ser cuestionada, encontramos casos en los que la reserva acaba siendo finalmente retirada y deja de surtir efecto.

Nuestro estudio también ha tomado en consideración otro elemento de gran valor a la hora de determinar el compromiso que adopta un Estado en relación con la protección de los Derechos Humanos. Nos referimos a la ratificación de los protocolos facultativos que tienen por objeto establecer un Comité como órgano de control en la aplicación del convenio, cuya competencia depende de la aceptación potestativa por parte del país.

En el caso particular de México, debemos señalar que si bien ha ratificado un buen número de protocolos facultativos, no siempre ha seguido las recomendaciones de Naciones Unidas en el sentido de ratificar o adherirse a la totalidad de los protocolos facultativos de aquellos convenios internacionales aceptados por este Estado. Al igual que ocurría con los convenios antes mencionados, México tampoco destaca en este contexto por la formulación de reservas o declaraciones interpretativas a estos protocolos.

De ahí que podamos concluir que, aunque la práctica convencional de México en materia de reservas resulta indicativa de una actitud favorable de este Estado hacia los convenios internacionales de protección de los Derechos Humanos, en la medida en que este país no ha aceptado la competencia de todos los órganos de control de la aplicación de estos instrumentos, resulta difícil evaluar la aplicación a nivel interno de estos

Asamblea General de las Naciones Unidas el 25 de mayo de 2000, Diario Oficial, Secretaría de relaciones internacionales, de 3 de abril de 2013. 
convenios y, por consiguiente, de su nivel de compromiso con las normas internacionales en la materia.

\section{Bibliografía.}

- ALIJA FERNÁNDEZ, R.A. y BONET PÉREZ, J. (2016), El control de los Tratados Internacionales de Derechos Humanos: realidad y límites. Una aproximación a la labor de los órganos de expertos desde la perspectiva de la efectividad de los derechos económicos, sociales y culturales. Barcelona: Atelier libros jurídicos.

- CARDONA LLORENS, J. (2019) "The legal value of the views and interim measures adopted by united nations treaty bodies (a response to the opinions of E. Jimenez Pineda, C. Jiménez Sánchez and B. Vázquez Rodríguez)”, Spanish yearbook of international law, vol. 23: pp. 146-163.

- CARRILlO SALCEDO, J.A. (1996), “Cours général de Droit International Public: Droit International et Souveraineté des Etats", Recueil des Cours de l'Académie de Droit International, vol. 257: pp. 217.

- CHAUMONT, C. (1970), "Cours général de droit international public", Recueil des Cours, vol. 129: pp. 333-528.

- GARCÍA RICO, E.M. (2010), “Algunas reflexiones en torno a la práctica española sobre reservas a los tratados de Derechos Humanos de ámbito universal", Colección Escuela Diplomática, n¹6, vol. 2: pp. 235-247.

- HATHAWAY, O.A., (2002) "Do Human Rights Treaties Make a Difference?", The Yale Law Yournal, Faculty Scholarship Series, Vol. 111, Paper 839: pp. 1935-2042.

- HORN, F. (1988), Reservations and Interpretative Declarations to Multilateral Treaties. La Haya: T.M.C. Asser Instituut, Institute for International and European Law.

- IMBERT, P.-H. (1979), Les réserves aux traités multilatéraux. Evolution du droit et de la practique depuis l'avis consultatif donné par la Cour internationale de Justice le 28 mai 1951. Paris: Editions A. Pedone.

- JIMÉNEZ SÁNCHEZ, C. (2019), "Human Rights Committees: Their nature and legal relevance in Spain", Spanish yearbook of international law, vol. 23: pp. 104128.

- LÓPEZ HURTADO, C. (2001), “¿Un régimen especial para los tratados de Derechos Humanos dentro del Derecho Internacional? Reservas y denuncias de tratados de Derechos Humanos", Anuario Mexicano de Derecho Internacional, vol. I, pp. 247-289.

- MARÍN AÍS, J.R. (2015), "Reservas incompatibles con el objeto y fin de la convención para la eliminación de todas las formas de discriminación contra la mujer. 
El caso de los estados islámicos", Revista Electrónica de Estudios Internacionales, núm. 29: pp. 63.

- MILANOVIC, M. y SICILIANOS, L.-A. (2013), "Reservations to traties: An Introduction", The European Journal of International Law, vol. 24, nº 4 : pp. 10551059 .

- PELLET, A. (2013), "The ILC Guide to Practice on Reservations to Treaties: A General Presentation by the Special Rapporteur", The European Journal of International Law, vol. 24, nº 4: pp. 1061-1097.

- QUEL LÓPEZ, J. (1991), Las reservas a los tratados internacionales (un examen de la práctica española). Bilbao: Servicio Editorial de la Universidad del País Vasco.

- REMIRO BROTONS, A. et al. (2010), Derecho internacional: curso general. Valencia: Tirant lo Blanch.

- RIQUELME CORTADO, R. (2004), Las reservas a los tratados: Lagunas y ambigüedades del Régimen de Viena. Murcia: Universidad de Murcia, Servicio de Publicaciones, Colección Estudios de Derecho.

- SOLARI YRIGOYEN, H. "Las reservas a los Tratados Internacionales de Derechos Humanos", Agenda Internacional, no8: pp. 72-85. 